\title{
Theory and FDTD simulations of an amorphous silicon planar waveguide structure suitable to be used as a surface plasmon resonance biosensor
}

\author{
Alessandro Fantoni* ${ }^{1,2}$, João Costa ${ }^{1,2}$, Miguel Fernandes ${ }^{1,2}$, Yury Vygranenko ${ }^{2}$, Manuela \\ Vieira ${ }^{1,2,3}$ \\ 1. ADEETC-ISEL-Instituto Politécnico de Lisboa, Lisbon, \\ 2. CTS-UNINOVA, Caparica, Portugal \\ 3. DEE-FCT-UNL, Caparica, Portugal \\ (*) e-mail: afantoni@deetc.isel.ipl.pt
}

Received: 05/11/2019 Accepted: 15/04/2020

DOI: 10.7149/OPA.53.2.51032

\begin{abstract}
:
In this paper we present our work concerning the design of a semiconductor waveguide structure to be used as a biosensor based on Surface Plasmonic Resonance effects (SPR). The proposed structure is a planar metaldielectric waveguide where the sensor operation is based on the coupling between the fundamental propagation TM mode and the surface plasmon excited at the outer boundary of the metal, which interfaces the sample medium. Gold and aluminium are the metals considered for the plasmonic coating, amorphous silicon and a reduced graphene oxide layer are considered for the waveguide structure. FDTD simulations of the proposed structure show a clear attenuation peak in the output power at the wavelength where the plasmonic resonance is excited.
\end{abstract}

Keywords: amorphous silicon, SPP, waveguide, plasmonic sensor

\section{REFERENCES AND LINKS / REFERENCIAS Y ENLACES}

[1] 0. Tokel, F. Inci, and U. Demirci, "Advances in plasmonic technologies for point of care applications," Chemical Reviews, vol. 114, no. 11. pp. 5728-5752, 2014.

[2] P. Yager, G. J. Domingo, and J. Gerdes, "Point-of-Care Diagnostics for Global Health," Annu. Rev. Biomed. Eng., vol. 10, no. 1, pp. 107-144, 2008.

[3] B. A. Prabowo, A. Purwidyantri, and K. C. Liu, "Surface Plasmon Resonance Optical Sensor: A Review on Light Source Technology," Biosensors, vol. 8, no. 3. 2018.

[4] B. Pačková, N. S. Lynn Jr, J. Slabý, H. Šípová and J. Homola. "A route to superior performance of a nanoplasmonic biosensor: consideration of both photonic and mass transport aspects." Acs Photonics, 5(3), 1019-1025, 2018.

[5] X. D. Hoa, A. G. Kirk, and M. Tabrizian, "Towards integrated and sensitive surface plasmon resonance biosensors: A review of recent progress," Biosensors and Bioelectronics, vol. 23, no. 2. pp. 151-160, 2007.

[6] F. C. Chien and S. J. Chen, "A sensitivity comparison of optical biosensors based on four different surface plasmon resonance modes," in Biosensors and Bioelectronics, 2004, vol. 20, no. 3, pp. 633642.

[7] J. Dostálek et al., "Surface plasmon resonance biosensor based on integrated optical waveguide," in Sensors and Actuators, B: Chemical, 2001, vol. 76, no. 1-3, pp. 8-12.

[8] S. Nayak, N. R. Blumenfeld, T. Laksanasopin, and S. K. Sia, "Point-of-Care Diagnostics: Recent Developments in a Connected Age," Analytical Chemistry, vol. 89, no. 1. pp. 102-123, 2017.

[9] H. Shafiee et al., "Paper and flexible substrates as materials for biosensing platforms to detect multiple biotargets," Sci. Rep., vol. 5, 2015.

[10] E. Wijaya et al., "Surface plasmon resonance-based biosensors: From the development of different SPR structures to novel surface functionalization strategies," Current Opinion in Solid State and 
Materials Science, vol. 15, no. 5. pp. 208-224, 2011.

[11] T. Aihara et al., "Monolithic integration of surface plasmon detector and metal-oxidesemiconductor field-effect transistors," IEEE Photonics J., vol. 5, no. 4, 2013.

[12] H. Hu, X. Zeng, D. Ji, L. Zhu, and Q. Gan, "Efficient end-fire coupling of surface plasmons on flat metal surfaces for improved plasmonic Mach-Zehnder interferometer," J. Appl. Phys., vol. 113, no. 5, 2013.

[13] J. Dostálek, H. Vaisocherová, and J. Homola, "Multichannel surface plasmon resonance biosensor with wavelength division multiplexing," in Sensors and Actuators, B: Chemical, 2005, vol. 108, no. 12 SPEC. ISS., pp. 758-764.

[14] R. Takei, "Amorphous Silicon Photonics," in Crystalline and Non-crystalline Solids, 2016.

[15] G. Cocorullo, F. G. Della Corte, R. De Rosa, I. Rendina, A. Rubino, and E. Terzini, "Amorphous siliconbased guided-wave passive and active devices for silicon integrated optoelectronics," IEEE J. Sel. Top. Quantum Electron., vol. 4, no. 6, pp. 997-1001, 1998.

[16] A. Harke, M. Krause, and J. Mueller, "Low-loss singlemode amorphous silicon waveguides," Electron. Lett., vol. 41, no. 25, pp. 25-26, 2005.

[17] R. Sun et al., "Transparent amorphous silicon channel waveguides with silicon nitride intercladding layer," Appl. Phys. Lett., vol. 94, no. 14, 2009.

[18] F. G. Della Corte and S. Rao, "Use of amorphous silicon for active photonic devices," IEEE Trans. Electron Devices, vol. 60, no. 5, pp. 1495-1505, 2013.

[19] A. Khanna et al., "Amorphous silicon optical waveguides and Bragg mirrors," in Silicon Photonics and Photonic Integrated Circuits, 2008, vol. 6996, p. 699605.

[20] S. Kageyama, M. Akagawa, and H. Fujiwara, "Dielectric function of a-Si:H based on local network structures," Phys. Rev. B - Condens. Matter Mater. Phys., vol. 83, no. 19, 2011.

[21] A. Fantoni, P. Lourenco, and M. Vieira, "A model for the refractive index of amorphous silicon for FDTD simulation of photonics waveguides," in Proceedings of the International Conference on Numerical Simulation of Optoelectronic Devices, NUSOD, 2017, pp. 167-168.

[22] Sharma, P., Tuteja, S. K., Bhalla, V., Shekhawat, G., Dravid, V. P., \& Suri, C. R. "Bio-functionalized graphene-graphene oxide nanocomposite based electrochemical immunosensing", Biosensors and Bioelectronics, 39(1), 99-105, 2013

[23] F. Xu et al., "Complex refractive index tunability of graphene at $1550 \mathrm{~nm}$ wavelength," Appl. Phys. Lett., vol. 106, no. 3, 2015.

[24] Z. Lu and W. Zhao, "Nanoscale electro-optic modulators based on graphene-slot waveguides," J. Opt. Soc. Am. B, vol. 29, no. 6, p. 1490, 2012.

[25] Fantoni, A., Costa, J., Fernandes, M., Vygranenko, Y., \& Vieira, M. "A simulation analysis for dimensioning of an amorphous silicon planar waveguide structure suitable to be used as a surface plasmon resonance biosensor", Fourth International Conference on Applications of Optics and Photonics (Vol. 11207, p. 112070A). International Society for Optics and Photonics, 2019.

[26] K. M. McPeak et al., "Plasmonic films can easily be better: Rules and recipes," ACS Photonics, vol. 2, no. 3, pp. 326-333, 2015.

[27] H. S. Skulason, P. E. Gaskell, and T. Szkopek, "Optical reflection and transmission properties of exfoliated graphite from a graphene monolayer to several hundred graphene layers," Nanotechnology, vol. 21, no. 29, 2010.

[28] A. B. Djuriši and E. H. Li, “Optical properties of graphite," J. Appl. Phys., vol. 7404, no. 10, pp. 74047410, 1999.

[29] J. Q. Xi et al., "Optical thin-film materials with low refractive index for broadband elimination of Fresnel reflection,” Nat. Photonics, vol. 1, no. 3, pp. 176-179, 2007.

[30] P. Songkeaw, K. Onlaor, T. Thiwawong, and B. Tunhoo," Reduced graphene oxide thin film prepared by electrostatic spray deposition technique", Materials Chemistry and Physics, 226, 302308, 2019.

\section{Introduction}

Detection and molecular profiling of small biomolecules is technically challenging. Typically, conventional isolation methods require extensive post-labelling procedures, which are unreasonable on a clinical setting. The ELISA method, one of the most effective approaches in current use, entails considerable processing complexity (sample transportation, manual preparation steps, skilled technicians, several hours or days to obtain results) which leads to a preclusion for primary care settings [1]. On the other hand, global health policies move in the direction of Point-Of-Care testing [2] which can only be achieved 
with the development of new devices to detect and measure the concentration of proteins in small volumes of biologic fluids. Within this context of application and with this critical demand in mind, Surface Plasmon Resonance (SPR) sensors have received an enormous attention and found extensive use for chemical and biological applications [3]. Sensors based on plasmonic resonance can achieve higher sensitivity when compared with other evanescent wave sensors. This is because the resonance condition is highly dependent on the local refractive index near the metal surface, where the target biomarkers bind to immobilized antibodies. In addition to improved sensitivity, SPR sensors can also be made at the nanoscale thus providing a route to miniaturization [4]. They offer the potential to move proteomic biology into the clinical setting as a routine diagnostic procedure and surpass the technical challenge of conventional methods. One fundamental point in the design of the SPR sensor is to fine-tune the conditions for the existence of coupling between the excitation field and the Surface Plasmon Polariton (SPP) that propagates at the interface of the metal and the sample medium [5]. Targeting the optimization of this key point, a large panorama of different structure configurations has been proposed in the recent past, mainly based on different schemes for SPP generation and for sensor interrogation methods [6], allowing to produce highly sensitive sensors including miniaturized structures based on optical waveguides [7]. Nevertheless, there is still a strong demand for providing new cost-effective solutions which could improve access to healthcare and the diagnosis of infectious and chronic diseases [8]. There is much interest in the development of new strategies for bringing the excellent detection properties of SPR sensors to the playground of low-cost devices and materials [9]. The key element to transform a SPR structure into a biosensor is the surface functionalization with antibodies able to trap specific biomarkers [10]. From this point of view, the functionalization method must be simple and affordable to implement.

At the same time, to minimize the cost of the overall sensor, its fabrication must be integrated with standard microelectronic production technologies [11]. In addition, it should include a simplified, while still efficient, light coupling method, without the need of including external optical prisms or highly precise lithographic steps for grating or slits imprinting [12]. Also, another requirement is to develop solutions that fit a multiplexing approach [13] for parallel multi-proteomic analysis, enabling the creation of lab-on-chips for Point-Of-Care applications.

Hydrogenated amorphous Silicon (a-Si:H) is an attractive material for being used in photonic components such as, directional couplers, interferometers, filters and ring resonators [14]. State of the art good quality a-Si:H can be deposited by Pressure Enhanced Chemical Vapor Deposition (PECVD) at low temperatures (between 200 and $400^{\circ} \mathrm{C}$ ), making the deposition of this material compatible with back-end CMOS processing. In fact, in recent years, a-Si:H has been reported by many authors as a possible candidate for being used in mass production of photonics circuits [15-19]. The electronic optical properties of a-Si:H films are strongly influenced by the deposition technique and conditions. Optimal refractive index of a$\mathrm{Si}: \mathrm{H}$, measured by reflectometry [20], is 3.65 at $1550 \mathrm{~nm}$ which is a higher value than the crystalline silicon counterpart, but presence of defects and dangling bonds in the lattice results in high density of localized states at energies below the energy gap, producing photon absorption in the near IR range at telecommunication wavelengths. Hydrogen can passivate defects and its concentration is strongly dependent, among other factors, on the deposition temperature, determining the sub-gap absorption coefficient and producing small variations of the amorphous silicon optical functions. We consider the dependence of the material characteristics on the specific deposition conditions, using the GUTL model [21] to describe the a-Si:H optical properties, extending the standard Urbach-Tauc-Lorenz model to the infrared part of the spectrum.

In this work we propose a functionalized surface based on reduced graphene oxide rGO which allows the improvement of the biocompatibility, a cost reduction in terms of sensor production and a great improvement in its efficiency. Combining good optical properties with chemical functionality, graphene oxide has been reported as an optimal choice for compatible bio-interface development using specific antibody functionalization [22] Also, according to the measurements obtained for characterization of graphene based electro-optical modulators, the complex reflective index in the infrared range of graphene-based materials can be tuned by means of the application of an external electrical bias [23, 24]. Such feature opens the possibility of future device optimization by tuning the plasmonic modes for the waveguide SPR sensor.

This paper reports a modal analysis of the a-Si:H plasmonic waveguide which is at the basis of the proposed sensor device [25]. In addition, it extends our previous work to include FDTD simulations of the proposed plasmonic structures. 


\section{2. SPP Excitation and Optical Properties of Materials}

\section{2.a. Material Properties}

SPPs are a quasi-particle representing the electromagnetic excitations at a metal/dielectric interface. Under specific conditions, the evanescent waves become coupled to the surface collective oscillations of the free electrons, supporting their propagation at the metal-dielectric interfaces. In a simplified manner, it is possible to consider SPPs as hybrid modes comprising the features of both the propagating EM waves in dielectrics, which are transverse waves, and free electron plasma oscillations in metals, which are longitudinal. In TE modes there is no electric field in the direction of propagation so they cannot be coupled to the longitudinal electron oscillations in metals, only TM modes can couple to SPPs waves. The mode propagation constant ksp is given by the following expression, as a function of the optical properties of the materials ( $\varepsilon \mathrm{m}$ and $\varepsilon_{\mathrm{d}}$ for the metal and the dielectric complex permittivity, respectively), and in last instance as a function of the angular frequency $(\omega)$ :

$$
k_{s p}=\frac{\omega}{c} \sqrt{\frac{\varepsilon_{m}(\omega) \varepsilon_{d}(\omega)}{\varepsilon_{m}(\omega)+\varepsilon_{d}(\omega)}}
$$

The mode propagation constant $k_{s p}$ is a complex number and the excitation of a SPP requires the existence of a positive real part of $k_{s p}$. Such a necessary condition is verified in a large variety of metal/dielectric combinations in the visible or in the infrared range. The imaginary part of $k_{s p}$ determines the propagation length of the SPP, and if the $\operatorname{Im}\left(k_{s p}\right)$ assumes relatively large values, practical applications of the SPP become unsuitable. To analyse the conditions for SPP excitation, we have used the values for the refractive index of gold and aluminium taken from the literature [26], while for amorphous silicon our analysis uses the GUTL model. The complex refractive index of gold, aluminium and amorphous silicon is reported in Fig.1. We also consider the introduction of a thin layer of reduced graphene oxide (rGO) that will be acting in two ways, supporting the biological functionalization and allowing the SPP wavelength tuning. The refractive index of rGO is highly dependent on the deposition conditions and the layer thickness [27] allowing also the possibility of electrical tunability [24]. We have considered in our simulation tests, as an approximation of the refractive index of a rGO thin layer, the refractive index of graphite [28], with and without imaginary extinction coefficient.

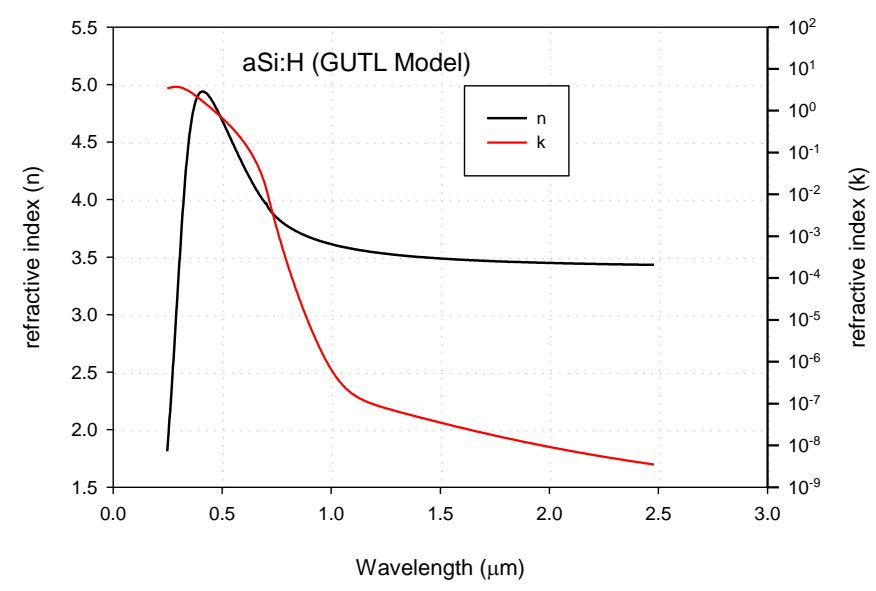

Fig.1 Real and imaginary parts of the refractive index obtained with the GUTL model [21] of a-Si:H, as used in the simulation.

\section{2.b. Modal Analysis}

Ideally the device structure should be simple for low-cost of production avoiding the requirement for complex lithographic processes based on multistep masks collimation. Among the different ways that can be used to excite the SPPs, our choice was to use slab wave coupling, where a waveguide mode propagates until encountering a region covered by a thin metal film, and by penetrating through the metal film couples with a SPP mode at the external metal interface. This coupling effect is only possible when the real part of the effective refractive index of the waveguide mode and the SPP mode are equal. Combining equation (1) with the theoretical dispersion curves of the fundamental TM mode of the a-Si.H slab 
waveguide, where the dielectric cladding on one side was replaced by the metal it is possible to determine the wavelength that fits the coupling condition, or in other words which is able to excite the desired SPP. A sketch of the sensor structure under consideration is reported in Figure 2. An a-Si:H waveguide deposited on $\mathrm{SiO}_{2}$ substrate transports its fundamental propagation TM mode, entering in the region covered by a thin metal layer $(50 \mathrm{~nm})$. If the SPP excitation conditions are fulfilled, part of the propagating EM energy is lost, and this can be detected at the waveguide output by a photodetector. The operation wavelength for the SPP excitation can be calculated by comparing the effective refractive index of the SPP with the effective index of the fundamental TM mode in the waveguide. The wavelength where is possible to excite an SPP is represented by the intersection point between the real parts of the effective refractive index of the SPP and the TM mode.

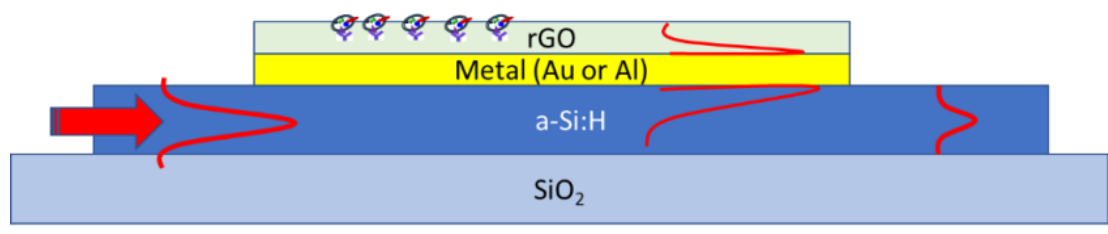

Figure 2. Vertical cut of the sensor device multiplanar structure. Light propagates into the waveguide and, when the right conditions are satisfied, excites an SPP on the two gold surfaces. The condition for the SPP generation is changed when the selective antibodies trap the target biomarker on the gold surface. When the SPP are generated, the intensity of the output light becomes attenuated.

Figure 3 reports the analysis based on the calculated effective refractive index for a simple structure based on a a-Si:H waveguide covered by a thin gold layer. We have considered the generation of an SPP at the gold surface covered by water, representing the sample medium containing the analytes. As can be seen, there is no useful wavelength for exciting the SPPs. An analogous situation is found if the gold layer is replaced by aluminium. Two different approaches can be considered to overcome this scenario: changing the substrate in order to decrease the value of the waveguide mode effective index, or replacing the material supporting the analyte to increase the SPP effective index. It has been demonstrated that electron beam deposition of $\mathrm{SiO}_{2}$ with very low refractive index (less than 1.1) is possible [29]. The use of this material as a waveguide substrate would allow the compatibility of a-Si:H waveguide modes with the SPP mode at the gold/water interface. The introduction of a thin layer of rGO between the metal and the sample medium would fit the second strategy. In Figure 4 we report the fundamental TM mode dispersion curves for both these alternative configurations. In both cases it is possible to find an operating wavelength that can be used to excite the SPPs. The first approach shows a lower absorption for plasmons and represents a very interesting alternative to improve the sensor structure. Nevertheless, as reported in [29], the deposition method of low index $\mathrm{SiO}_{2}$ is realized by oblique-angle deposition using electron-beam evaporation. This technique is not compatible with the low-cost requirements of our deposition system, which is based on the PECVD method.
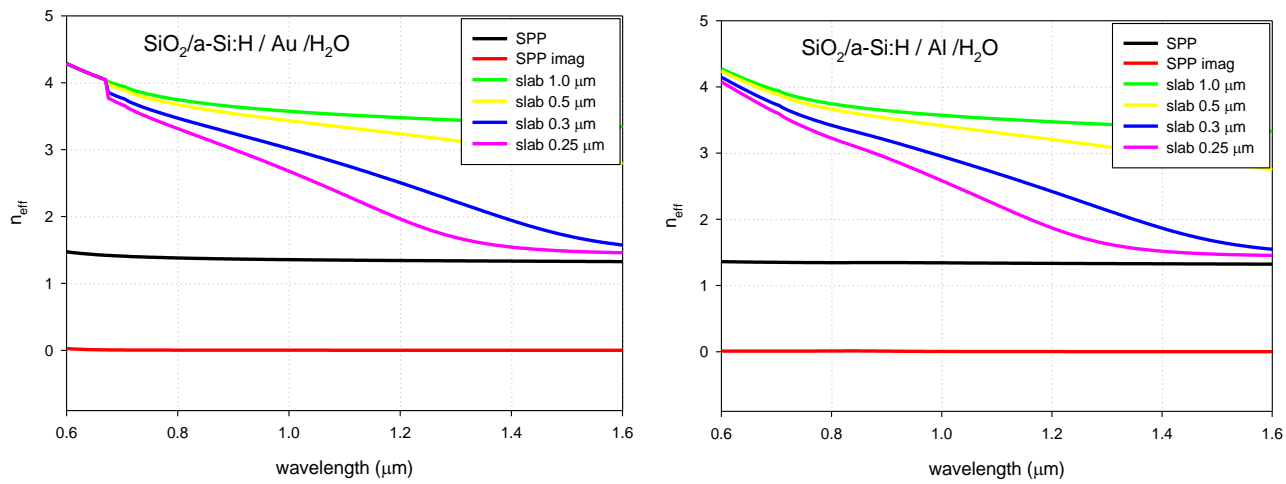

Figure 3 Effective refractive index for the SPPs compared with the fundamental TM mode in the a-Si:H waveguide. The black curve shows the real part of the SPP effective index, while the different plots refer to different thickness of the slab a-Si:H waveguide. 
The structure with the rGO treatment produces SPP with a high value of the imaginary part of the refractive index. The operating wavelength is found in a very interesting range, where the waveguiding material is transparent, crystalline silicon is still absorbing up to a wavelength of $1.1 \mu \mathrm{m}$. This combination allows the integration of an entirely silicon-based system of waveguide and photodetector.
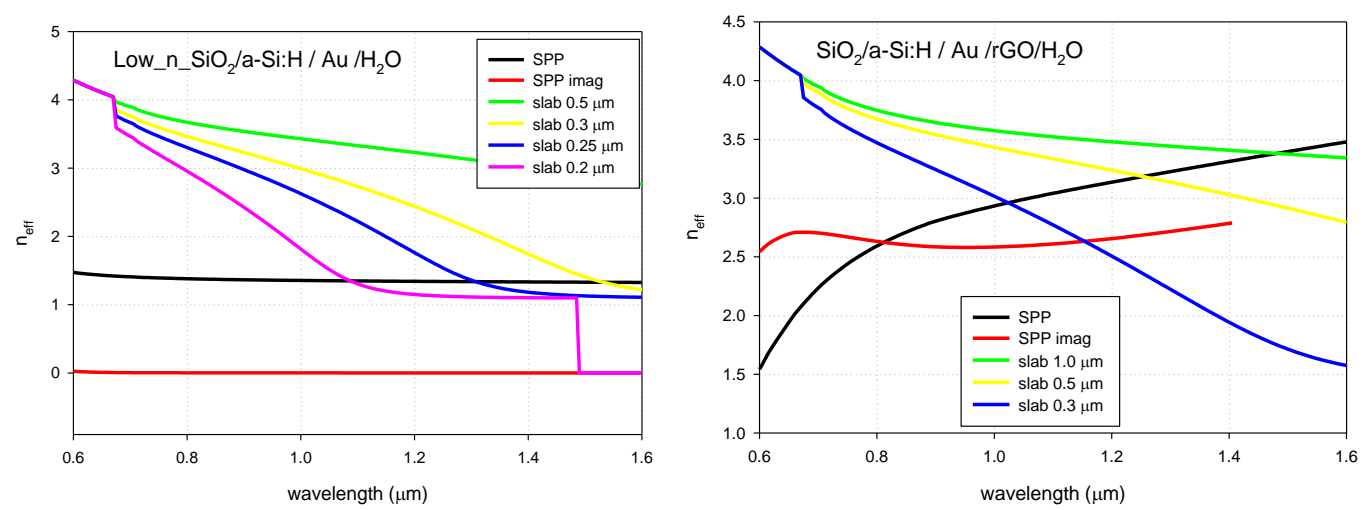

Figure 4 Effective refractive index for the SPPs compared with fundamental TM mode of the aSi waveguide. The structure on the left uses a low index $\mathrm{SiO}_{2}$ as a substrate, while the structure on the right uses rGO on top of the gold layer

A similar outcome can be observed in Figure 5, where the analysis is based on a non-dispersive model for the rGO covering an aluminium layer. Again, the operating wavelength falls in a useful range. The presence of the rGO layer allows, through small changes of its thickness, wavelength tuning of the SPPs. This characteristic offers a very attractive possibility, as the operating wavelength of the device can be tuned in the range of low-cost commercial light sources. Several methods exist to fabricate rGO film from a graphene oxide precursor solution and while the layer thickness control is generally a difficult task, electrostatic spray deposition (ESD) is a process that has been reported to allow the layer thickness control through the substrate temperature [29]. In this technique, the film thickness decreases when the deposition is realized at higher substrate temperature. The required temperature range remains below $400{ }^{\circ} \mathrm{C}$ and it is compatible with materials deposited by PECVD.
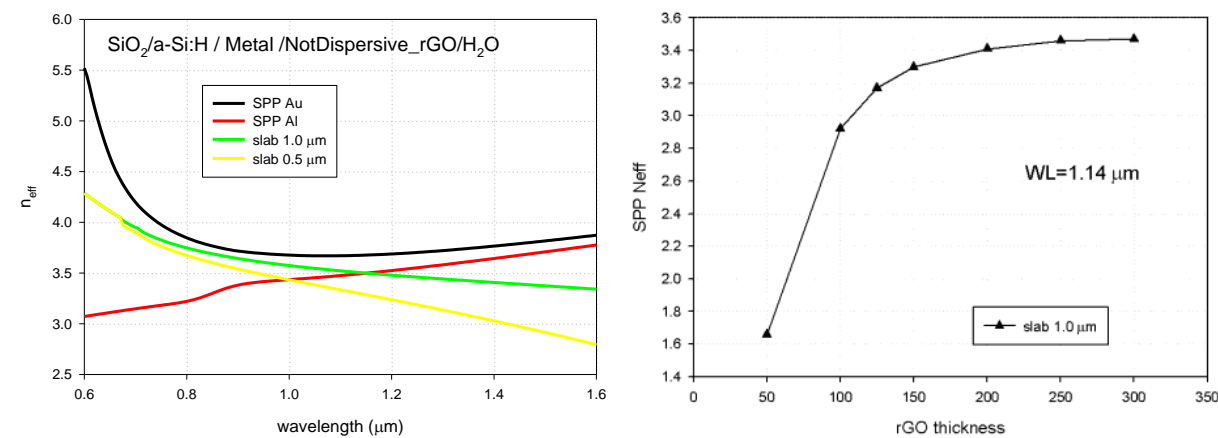

Figure 5. Effective refractive index for the SPPs compared with the fundamental TM mode of the aSi waveguide. The structure on the left uses standard $\mathrm{SiO}_{2}$ as a substrate, and non dispersive $\mathrm{rGO}$ on top of the metal layer (Au or Al). The plot on the right show the dependence of the SPP effective index on the rGO thickness.

In Figure 6 we have used numerical simulations based on FDTD method at 980 nm to study the transversal $\mathrm{H}$ field amplitude on the structures. In the $\mathrm{H}_{2} \mathrm{O} / \mathrm{rGO} / \mathrm{Al} / \mathrm{a}-\mathrm{Si}: \mathrm{H} / \mathrm{SiO}_{2}$ waveguide structure we can see that the SPP is excited causing considerable attenuation of the field in the core region, as expected. We may also note that the light attenuation produced by the material sub-gap absorption is negligible in the near infrared and for a waveguide length limited to a few tenths of $\mu \mathrm{m}$. Therefore, in absence of the SPP generation, light propagates in the waveguide and reaches the output monitor. When the condition to excite the SPP is fulfilled, its dispersive properties cause the light not reaching the waveguide output. The sensor output reading is based on a wavelength scan, producing a minimum in the output power when the 
SPP is excited. The position of this wavelength minimum can be related to the refractive index of the analyte absorbed by the rGO. When the target biomarkers are immobilized by the selective antibodies, the medium refractive index suffers a change and the output minimum is shifted. Figure 7 reports the overlap power, calculated against the input power for two thickness of the slab a-Si:H waveguide. The minimum corresponds in both cases to the wavelength where the dispersive SPP is excited. The result agrees with the modal analysis presented in Figure 5. In the $0.5 \mu \mathrm{m}$ case, when the wavelength increases toward 1.1 $\mu \mathrm{m}$, the waveguide becomes too small and the propagation of light become less efficient, causing a decrease in the output intensity independently from the excitation of the SPPs. It can be also observed that in this latter case, the light power output is much lower than in the case with the 1.0 slab case. This can be explained by a fair optimization of the wavelength lengths. A multiparameter optimization of the structure dimensions should be carried on for determining the optimal configuration for the required wavelength range, but such a study is beyond the scope of this work.
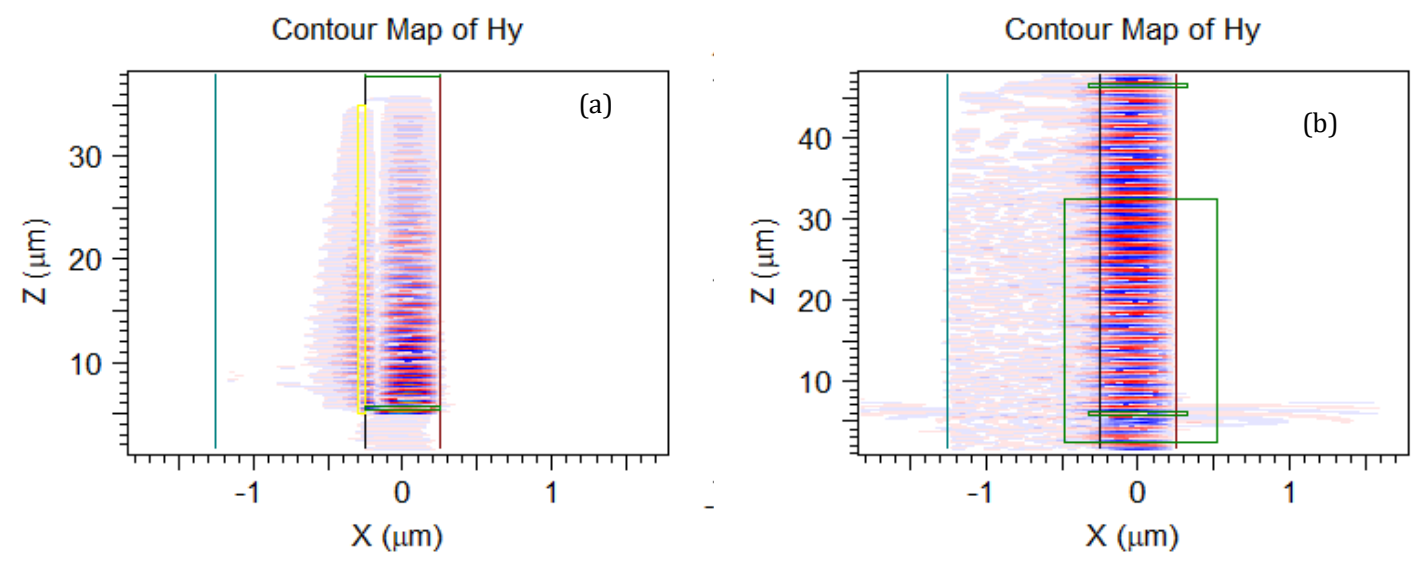

Figure 6. (a) FDTD simulation displaying the Hy field intensity in the $\mathrm{H}_{2} \mathrm{O} / \mathrm{rGO} / \mathrm{Al} / \mathrm{a}-\mathrm{Si}: \mathrm{H} / \mathrm{SiO}_{2}$ waveguide. The surface plasmon is present under coupling conditions. (b) In this case the metal interface is absent so there is no surface plasmon and the field intensity is less attenuated.
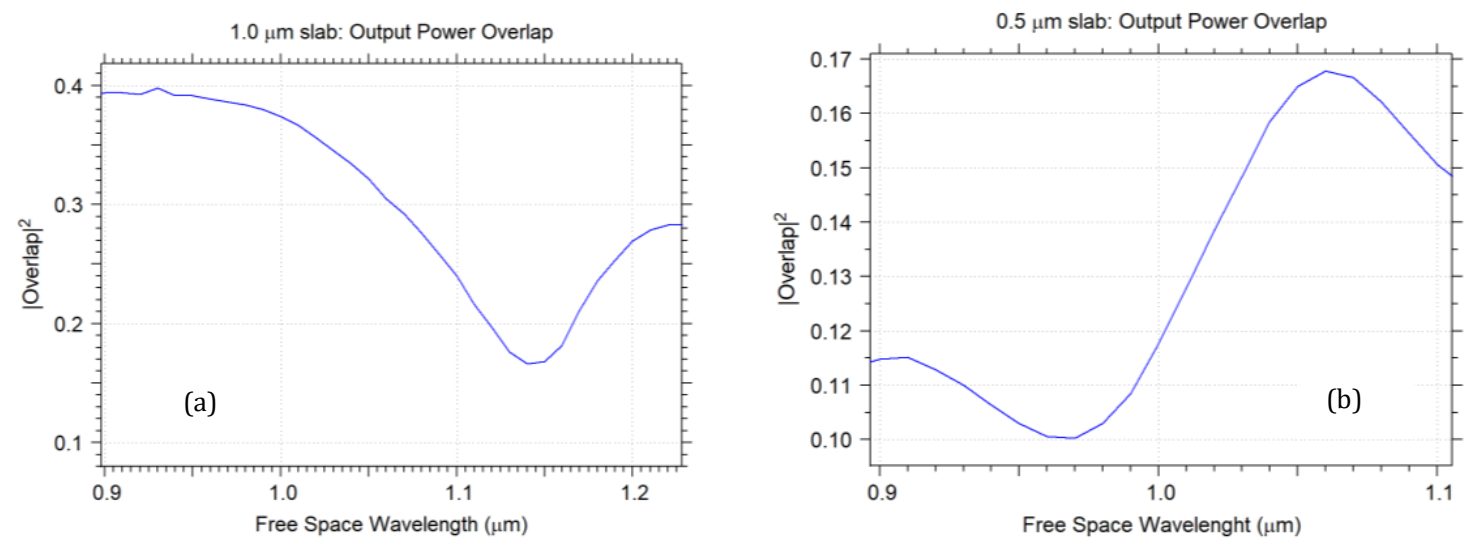

Figure 7. FDTD simulation displaying the output power in the $\mathrm{H}_{2} \mathrm{O} / \mathrm{rGO} / \mathrm{Al} / \mathrm{a}-\mathrm{Si}: \mathrm{H} / \mathrm{SiO}_{2}$ structure. Waveguide slot has $1.0 \mathrm{~mm}$ (a) and $0.5 \mathrm{~mm}$ (b). In both cases the minimum corresponds to the excitation of a dispersive SPP.

\section{Conclusions}

The conditions for coupling the fundamental TM mode of a-Si:H waveguide to a SPP were studied using different cladding materials targeting the design of a low-cost SPR device for biosensing applications. Low cost materials like amorphous silicon can be used to waveguide light in the infrared range, offering the opportunity for an integrated all silicon-based structure for waveguide and photodetector. The 
introduction of the reduced graphene oxide as a cover layer for the metal/ analyte interface offers the possibility of tuning the device's wavelength coupling condition. The FTDT simulations of the novel structure show that excitation of the SPP is achievable even at $980 \mathrm{~nm}$, assuming water as the sample medium. The replication of the sensing mechanism at a common operation wavelength and for a representative refractive index of the sample medium opens the door to the development of SPR biosensors based on amorphous silicon waveguides. Moreover, while at $980 \mathrm{~nm}$ a-Si:H is transparent, nanocrystalline silicon (nc-Si:H) absorbs light and thus can be used as a photodetector material opening up the possibility for a fully integrated silicon device.

\section{Acknowledgements}

Research supported by EU funds through the FEDER European Regional Development Fund and by Portuguese national funds by FCT -Fundação para a Ciência e a Tecnologia with projects PTDC/NANOPT/31311/2017, UID/EEA/00066/2019 and by project IPL/2018/aSiPhoto_ISEL. Part of this work was presented at the IV International Conference on Applications in Optics and Photonics (AOP 2019, paper 28). 